\title{
Erratum to: Metaphysics, prescription and methodological disagreement
}

\section{A comment on Mathias Frisch's Causal reasoning in physics}

\author{
Alexander Reutlinger ${ }^{1} \cdot$ Phyllis Illari $^{2}$. \\ Andreas Hüttemann ${ }^{3}$ - Mathias Frisch ${ }^{4}$
}

\section{Erratum to: Metascience (2015) 24:351-372 DOI 10.1007/s11016-015-0011-3}

This contribution should read:

Symposium

Mathias Frisch: Causal Reasoning in Physics

Metaphysics, prescription and methodological disagreement: A comment on Mathias Frisch's Causal reasoning in physics

by Alexander Reutlinger

Why reasoning?: A discussion of Matthias Frisch's: Causal reasoning in physics by Phyllis Illari

On adding causal structure: Comment on Mathias Frisch's Causal reasoning in physics by Andreas Hüttemann

Replies

by Mathias Frisch

The online version of the original article can be found under doi:10.1007/s11016-015-0011-3.

Alexander Reutlinger

Alexander.Reutlinger@1rz.uni-muenchen.de

Phyllis Illari

phyllis.illari@ucl.ac.uk

Andreas Hüttemann

ahuettem@uni-koeln.de

Mathias Frisch

mfrisch@umd.edu

1 Munich, Germany

2 Department of Science and Technology Studies, University College London, London, UK

3 University of Cologne, Cologne, Germany

4 University of Maryland, College Park, MD, USA 